\title{
Histogram Intersection based Image Retrieval Technique using Relevance Feedback
}

\author{
Tudor Barbu, Mihaela Costin, Adrian Ciobanu \\ Institute of Computer Science, Romanian Academy, Iaşi branch \\ Carol I 22A, Iaşi, Romania \\ tudbar@iit.tuiasi.ro, mccostin@ iit.tuiasi.ro, cibad@iit.tuiasi.ro
}

\begin{abstract}
This article provides a color-based image retrieval technique for RGB image databases. Our proposed CBIR system uses the query by example approach and a relevance feedback mechanism. Feature extraction process is performed by computing a global color histogram for each image. Feature vectors are compared using the histogram intersection difference metric, and a relevance feedback mechanism is used in the retrieval process.
\end{abstract}

Keywords: RGB image, image base, CBIR, color-based image retrieval, color histogram, feature vector, histogram intersection, query by example, relevance feedback

\section{INTRODUCTION}

We propose a content-based image retrieval system, using color features, in this work. Digital image retrieval, an important sub-domain of multimedia (information) retrieval [1], is digital image retrieval [2].

An image retrieval system is a computer based system for browsing, searching and extracting entities from large digital image database, Web sites or video sequences.

The various digital image retrieval methods are usually divided into two main categories: text-based and content-based retrieval methods. Text-based image retrieval contains the most traditional and common techniques of image retrieval, and uses image annotation.

Digital image annotation represents the process of assigning metadata in the form of captioning, keywords or descriptions to the images [3], and could be performed manually or automatically. Interactive image annotation is a time-consuming, laborious and expensive process. To address these drawbacks, a lot of research has been performed on automatic image annotation, the procedure by which a computer system automatically assigns the metadata to the images.

Content-based image retrieval (CBIR) performs the image retrieval task through analyzing the image content [4]. It represents the application of computer vision to the image retrieval problem and aims to avoiding the use of semantic descriptions and instead retrieves images based on their visual similarity to a user-supplied query image or user-specified image features. The image content is a combination of colors, shapes, textures, or any other information that can be derived from the image itself. Thus, various color based [5], texture based [6] and shape based retrieval methods [7] have been developed recently. We approach here the RGB color based retrieval domain only [6].

The retrieval system is characterized by a querying technique [4]. There are various types of user queries, such as: query by example, query by multiple example, semantic queries, query by image region, querying by direct specification of image features and multimodal queries respectively.

Many CBIR systems are based on relevance feedback mechanisms [8]. Our image retrieval system uses a query by example method and a relevance feedback scheme, which are described in the second chapter of this work.

In the next section we provide some color histogram based image feature vectors and a vector comparison method. Some experiments performed with the proposed retrieval technique are described in the third chapter, the paper ending with a conclusions section.

\section{IMAGE FEATURE EXTRACTION}

We present a color based feature extraction approach for RGB images in this section. So, let us consider an RGB image database, or collection, containing $n$ images: $\left\{I_{1}, . ., I_{n}\right\}$. We are going to retrieve images from this database, based on their color similarity $[6,9]$.

We compute a global color histogram for each image of the collection, and use it as a feature vector. The 1D color RGB histogram proposed by us uses an index given by the following linear combination :

$$
\operatorname{ind}(r, g, b)=\left\lceil\frac{r}{2^{N_{b}}}\right\rceil \cdot 256+\left\lceil\frac{g}{2^{N_{b}}}\right\rceil \cdot 16+\left\lceil\frac{b}{2^{N_{b}}}\right\rceil
$$

where $N_{b}$ is the number of bits per pixel and $[r, g, b]$ represents the current RGB combination.

Let us set $N_{b}=4$, to reduce the histogram dimension ( $N_{b} \times N_{b} \times N_{b}$ ) and the computation complexity. Thus, from equation (1) we obtain the following color histogram index: 


$$
\operatorname{ind}(r, g, b)=\left\lceil\frac{r}{16}\right\rceil \cdot 256+\left\lceil\frac{g}{16}\right\rceil \cdot 16+\left\lceil\frac{b}{16}\right\rceil
$$

Thus, for each image $I_{i}$, we compute its color histogram $H_{I_{i}}$, using the $\operatorname{ind}(r, g, b)$ value computed by relation (2), instead of $[r, g, b]$. Therefore, we will have the following relation:

$$
H_{I_{i}}(r, g, b)=H_{I_{i}}\left(\left\lceil\frac{r}{16}\right\rceil \cdot 256+\left\lceil\frac{g}{16}\right\rceil \cdot 16+\left\lceil\frac{b}{16}\right\rceil\right)=k
$$

for each color combination $(r, g, b)$.

We could consider this computed histogram, which has 4096 coefficients, as a feature vector for the current image, therefore we get:

$$
V\left(I_{i}\right)=H_{I_{i}}, \forall i \in[1, n]
$$

These feature vectors can be compared using many histogram comparison techniques which could be used as metrics. Let us mention Euclidean distance, histogram intersection and cosine or quadratic distances, that can be used for these purpose.

We choose to use a histogram intersection approach for our system. The formula of this distance is given by the following relation:

$$
\operatorname{histin}\left(H_{I}, H_{J}\right)=\sum_{t=0}^{4095} \frac{\min \left(H_{I}(t), H_{J}(t)\right)}{\max \left(H_{I}(t), H_{J}(t)\right)}
$$

Therefore, the distance between two consecutive image feature vectors will be computed as:

$d\left(V\left(I_{i}\right), V\left(I_{j}\right)\right)=\operatorname{histin}\left(H_{I_{i}}, H_{I_{j}}\right), \forall i, j \in[1, n](6)$

The above equation will be further used in the retrieval process.

\section{RELEVANCE-FEEDBACK BASED MECHANISM}

As we have mentioned before, we provide a color-based image retrieval system, using the query by example interogation approach and the relevance feedback mechanism [4]. Any retrieval technique that is based on this querying method receives an example entity as an input that it will then base its search upon.

The relevance feedback engine is used by many retrieval systems [10]. The idea behind it is to take the results that are initially returned from a given query and to use information about whether or not those results are relevant to perform a new query.

The system proposed by us must perform the next retrieval operation: to find the desired image(s), from a large image base using an initial example [10]. The options for providing query images include: a preexisting image may be supplied by the user or chosen from a random set; the user draws an approximation of the desired image.

Let us propose the following CBIR color-based image retrieval algorithm. The example image is compared with those of the database first. The most relevant images, being similar by color content, are extracted from the base and displayed on a screen.

If the desired image can be found in this retrieved set, then the searching process ends. Otherwise, the relevance feedback scheme is used.

The most relevant image, in terms of color similarity, is interactively selected from the displayed image set and becomes the new input. The retrieval process performs these operations at each step, until a stopping condition is finally met.

We set as the retrieval process ending point, the moment when the current query image becomes more similar to the desired image than all images extracted and displayed in that step. That image can be considered the desired one, or no image from the collection is considered acceptable for the user.

The feature detector and the retrieval mechanism represent the main devices of our color-based image retrieval system. Thus, at each step, the retrieval device receives a query example image $I$. It is send to the feature extractor device, where the feature vector $V(I)$ is calculated.

Thus, we obtain the feature set $\left\{V\left(I_{1}\right), . ., V\left(I_{n}\right)\right\}$, corresponding to the images of the database. The most relevant $K$ images are retrieved from the image base, using the following algorithm.

One computes $d_{i}=d\left(V\left(I_{i}\right), d(V(I))\right.$, with $i \leq n$, then sort the distance value set $D=\left\{d_{i}\right\}_{i=1, n}$ in the ascending order. If the obtained set is $\left\{d_{n(i)}^{i}\right\}_{i=\overline{1, n}}, n(i)$ being the order of $d_{n(i)}^{i}$ in $D$, then the most relevant images for $I$ are $\left\{I_{n(1)}, \ldots, I_{n(K)}\right\}$. They are displayed on the screen to be visualized by the user.

If the established stopping condition is satisfied, then the retrieval process ends. Otherwise, the user selects a new input query $I \in\left\{I_{n(i)}\right\}_{i \in[1, K]}$, from that screen. The searching and extracting procedure repeats in the same way until the ending condition is reached.

\section{EXPERIMENTS}

We have performed many experiments, testing our CBIR color based image retrieval system on various large image datasets. We have got satisfactory retrieving results from all these tests.

For space reasons, we will present a short retrieval example here. So, let us consider an image base containing several hundreds digital RGB color images.

We set $K=6$, the number of relevant images retrieved 
and displayed at each step. Suppose we want to retrieve from the respective database, images containing historical vestiges. Therefore, we choose the following input query image:

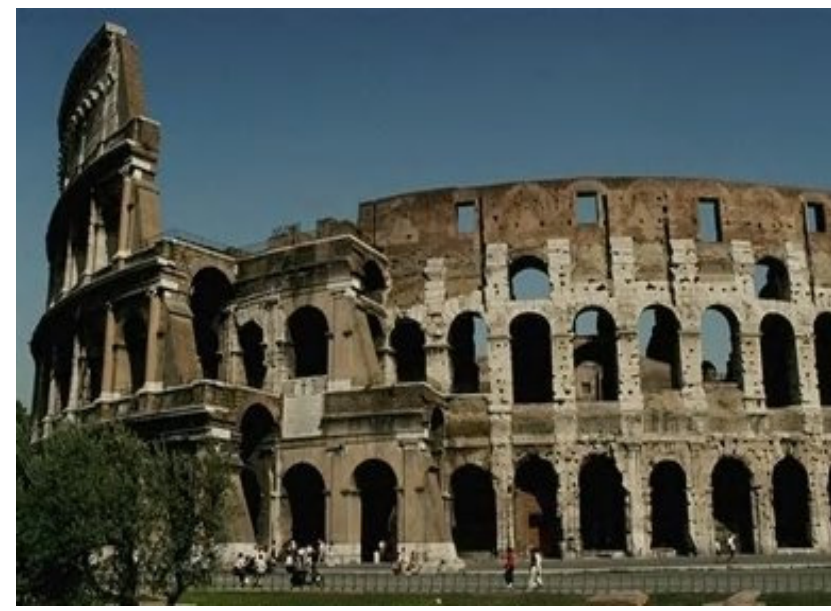

Figure 1. Example query image

The retrieved images, using this initial input, are those represented in Figure 2.

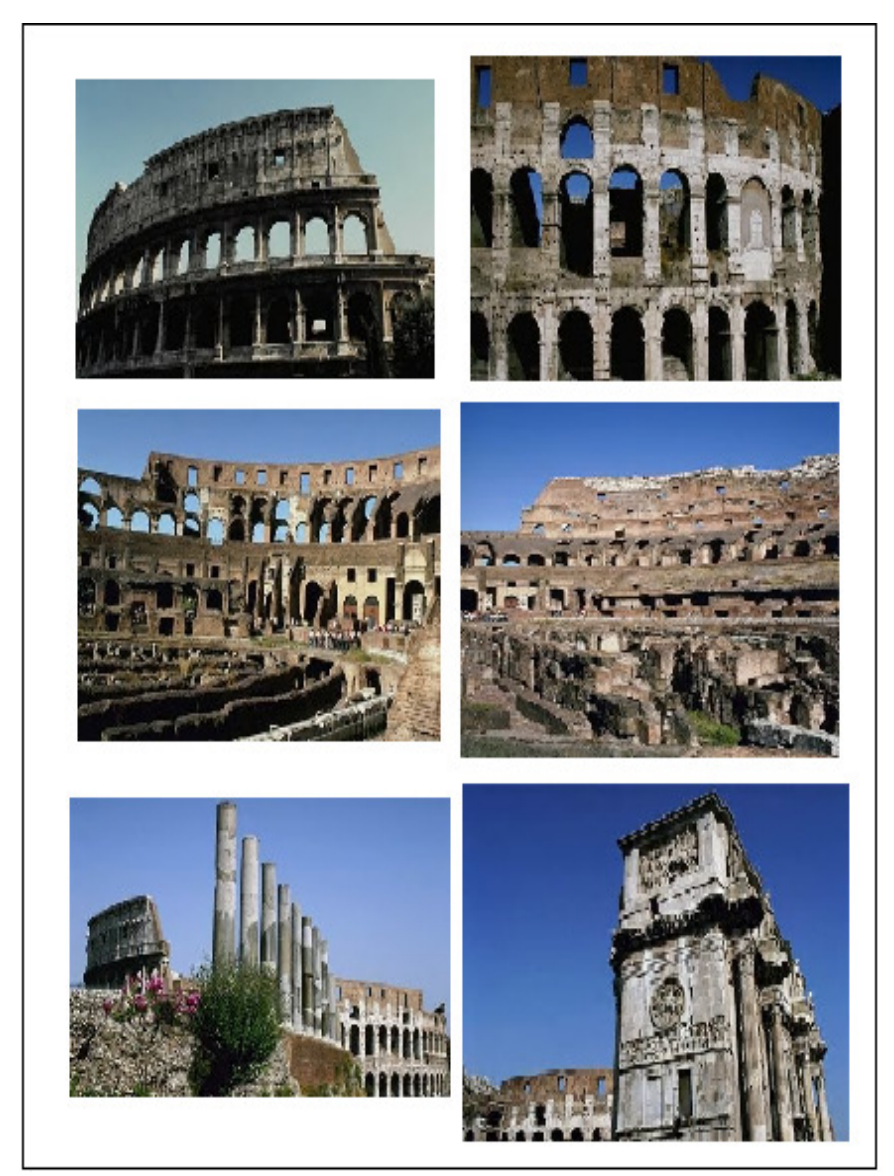

Figure 2. Retrieved images
The retrieved images are displayed in the descending order of their similarity to the input, as one can see from the figure. The retrieval process could continue if we selected one of the retrieved images as an input and apply the relevance feedback.

All the extracted color images represent vestiges, but if we choose the last image for relevance feedback another type of historical buildings will be retrieved.

\section{CONCLUSIONS}

A image retrieval technique using color characteristics has been proposed in this article. We have computed histogram-based feature vectors and considered a special difference metric, namely histogram-intersection, to compare them.

We also have provided a relevance feedback based retrieval procedure and a query by example interogation method. Many experiments have been performed, satisfactory results being obtained using this color-based CBIR system.

It is important that at each step, the retrieved images, displayed on the screen are similar by color with the input image. Unfortunately, color similarity does not represent always the same thing as the semantic similarity. It is possible to get images having identical color histograms but totally different semantics.

Because the digital image retrieval domain is closely related to image indexing [11], our future research will focus on obtaining some indexing techniques, using color features, that could facilitate the retrieval operations. Other content-based image retrieval fields, like shape-based and texture-based retrieval, will be approached as well.

\section{REFERENCES}

[1] M. Lew et al., "Content-based Multimedia Information Retrieval: State of the Art and Challenges", Phil. Trans. Roy. Soc. London, vol. A247, pp. 529-551, April 1955

[2]. R. Datta, D. Joshi, J. Li, J. Z. WANG, "Image Retrieval: Ideas, Influences, and Trends of the New Age", ACM Computing Surveys 40 (2): pp. 1-60, April 2008, doi:10.1145/1348246.1348248.

[3]. M. Inoue, "On the need for annotation-based image retrieval", Workshop on Information Retrieval in Context, pp. 44-46, 2004.

[4]. J. Eakins, M. Grahalm, "Content-based Image Retrieval", JISC Technology Applications Programme Report 39, 1999.

[5]. J. Smith, S.-F. Chang, "Tools and techniques for color image retrieval", In Symposium on Electronic Imaging: Science and Technology Storage \& Retrieval for Image and Video Databases IV, volume 2670, San Jose, CA, February 1996. IS\&T/SPIE.

[6]. J. Smith, S.-F. Chang, "Texture classification and discrimination in large image databases", In Proceedings of the I.E.E.E. International Conference on Image Processing (ICIP-94), November 1994.

[7]. D. Dimov, "Fast, Shape Based Image Retrieval", In Proceedings of International Conference on Computer Systems and Technologies, CompSysTech'2003, 2003.

[8]. I. AAalsberg, "Incremental relevance feedback", Proceedings of the Fifteenth Annual International ACM SIGIR Conference on Research and Development in Information Retrieval, Copenhagen, pp. 11-22, 1992.

[9]. G. Pass, R. Zabih, "Histogram refinement for content-based image retrieval", in Proceedings of the 3rd IEEE Workshop on Applications of Computer Vision (WACV '96), pp.96, 1996.

[10]. T. Barbu, "Modelling Multimedia Information Systems" (in Romanian), at Romanian Academy Publishing House, 2006.

[11]. M. Swain, D. Ballard, "Color indexing, International Journal of Computer Vision", 7(1):11-32, 1991. 
